\title{
BALANCE OF PROINFLAMMATORY AND ANTI INFLAMMATORY MARKERS IN ROUTINE HEMODIALYZED PATIENTS (A STUDY OF END- STAGE RENAL FAILURE PATIENTS AT THE DR. SARDJITO HOSPITAL
}

\author{
Ira Puspitawati ${ }^{1}$, Purwanto AP ${ }^{2}$, Lisyani B. Suromo ${ }^{2}$ \\ ${ }^{1}$ Clinical Pathology Department, Medical Faculty of Gadjah Mada University/Dr. Sardjito Yogyakarta, Indonesia. E-mail: \\ ipuspitawati@yahoo.com ${ }^{2}$ Clinical Pathology Department, Medical Faculty of Diponegoro University/Dr. Kariadi Hospital \\ Semarang, Indonesia
}

\section{ABSTRACT}

Patients with End-Stage Renal Disease (ESRD) tend to have immune imbalance triggered by uremia and Hemodialysis (HD) procedures. Contact between dialysis membrane and blood will cause bio-incompatibility reactions inducing complement activation and production of Reactive Oxygen Species (ROS) as well as proinflammatory cytokines and acute phase protein such as C-Reactive Protein (CRP). Those immune response imbalances will lead to an immunocompromised condition. The objective of this study was to prove the correlation between inflammatory markers (IL-1 $1, \mathrm{IL}-6$ and C-reactive proteins) and its anti-inflammatory marker (IL10 ) in routine hemodialyzed patients. This is a cross-sectional observational study involving 90 subjects conducted at the Hemodialysis Installation of the Dr. Sardjito Hospital. The inclusion criteria of this study were patients who underwent routine HD procedures, aged between 18 and 65 years-old, having leukocytes count and albumin level within normal limit. The exclusion criteria of this study were patients with Acute Coronary Syndrome (ACS) and malignancies. Levels of IL-1B, IL- 6 and IL-10 were measured using Enzyme-Linked Immunosorbent Assay (ELISA), while CRP was measured using highly-sensitive CRP immunoturbidimetry. Statistical analysis was performed by Spearman test. This study results showed correlations between IL-1ßand IL-10 $(p=0.001, r=0.302), I L-6$ and IL-10 ( $p=0.001, r=0.418)$ and correlation between CRP and IL-10 ( $p=0.005$, $r=0.295)$. There were also correlations between IL-1 $\beta$ and IL-6 $(p=0.029, r=0.232), I L-6$ and CRP $(p=0.001$, $r=0.534)$, but no correlation was found between IL- $1 \beta$ and CRP $(p=0.431, r=0.073)$. All factors that trigger the secretion of proinflammatory cytokines will trigger the release of anti-inflammatory cytokines, the consequences of anti-inflammatory cytokine secretion will happen minutes after the release of inflammatory cytokines. This study showed that there were correlations between proinflammatory and anti-inflammatory cytokines. Further studies of polymorphism-related cytokines secretion are warranted.

Key words: End stage renal disease, hemodialysis, proinflammatory markers, anti-inflammatory markers

\section{INTRODUCTION}

Chronic Kidney Disease (CKD) is a major problem in most developed and developing countries. In the United States, currently, there is a total of $13 \%$ population with CKD. The prevalence of CKD continues to increase each year. The most potential feature of CKD is the End-Stage Renal Disease (ESRD) which needs a kidney replacement therapy. In the United States, the progressive rate of CKD stage from stage 3 and stage 4 to become ESRD (or CKD stage 5 ) is $1.5 \%$ per year. ${ }^{1}$ Patients with CKD and ESRD have higher morbidity, mortality and hospitalization risks compared with non-CKD patients.
This condition will cause a significant burden of illness for the population.

As ESRD is closely related with inflammation, one of its features is increased inflammatory markers, such as interleukin 1? (IL-1?]), IL-6 and CRP. That inflammation will worsen with Hemodyalisis (HD) procedures. Some hemodialysis-related factors that may cause the inflammation are interactions between blood and dialyzer membrane which will trigger some inflammatory mediators, such as C3, C5 complements and Platelet Activating Factor (PAF) which in turn will activate neutrophils to release some Reactive Oxygen Species 
products inside the dialysate will cross the dialyzer membrane to further reactivate neutrophils. ${ }^{2}$

Current studies related with inflammation on hemodialyzed patients in Indonesia are still limited. The balance condition between proinflammatory and anti-inflammatory markers also has not been fully explored in Indonesia yet, even though race has a very strong influence in the balance of proinflammatory and anti-inflammatory markers.

A study conducted by Brown et al., showed that race has a very strong influence on cytokines production. Previous studies showed that there was influence from gene polymorphism coding the IL-1? production that will define individual susceptibility to get the ESRD, such as IL-1?-5887, IL-3953, IL$1 \mathrm{RN}+8006$ and IL-1RN86bp VNTR polymorphisms. This polymorphisms distribution differed in some countries such as Europe and the United States., Some studies also showed that race has an influence to the interleukin-6 (IL-6) secretion. A study conducted by Brown et al., ${ }^{3}$ showed that race will strongly influence the IL- 6 production, in which IL- 6 production was found higher in African American compared with Caucasian people. ${ }^{3}$ C-reactive protein level was also influenced by race. White race was found to have a lower CRP level compared with the Black race. Hispanic and Asian races were found to have higher CRP levels. ${ }^{5} \mathrm{C}$ is one of the acute-phase proteins which can reflect both acute and chronic inflammatory conditions. A study in Indonesia setting was needed to figure out how the balance response was between proinflammatory and anti-inflammatory markers in its population. The goal of this study was to know the balance between proinflammatory and antiinflammatory markers in ESRD patients and to see the correlations between proinflammatory (IL-1 $\beta$, IL-6, $\mathrm{CRP}$ ) and anti-inflammatory markers in routine hemodialyzed patients at the Dr. Sardjito Hospital Yogyakarta.

\section{METHODS}

This is a cross-sectional study, involving a total of 119 subjects. The inclusion criteria of this study were patients aged between 18 and 65 years-old, having normal body temperature at the time of study (not febrile), HBsAg negative, total leukocyte count and albumin level between normal limit range. The exclusion criteria were patients with Acute Coronary Syndrome (ACS) or malignancies and acute infections.
Three $\mathrm{mL}$ of EDTA blood were taken for routine blood assessment and $3.5 \mathrm{~mL}$ of non-anticoagulated blood were taken for proinflammatory markers (IL$1 \beta, I L-6, C R P)$ and anti-inflammatory marker (IL-10) assessments from patients who have fulfilled the inclusion and exclusion criteria of this study. The IL$1 \beta$, IL-6 and IL-10 levels were measured using ELISA method with reagents from e-Bioscience and were calculated using a microplate reader Biorad tools Imark series and CRP level was measured using Cobass 6000 with high sensitive-CRP immunoturbidimetry method. Other laboratory assessments were total leucocyte count and differential leukocyte count to exclude infections and BUN, creatinine and albumin levels. Other data that had correlation with risk factors, previous illness history and previous medication history were obtained using history and medical records tracking.

\section{RESULT AND DISCUSSION}

A total of 90 subjects who had fulfilled the inclusion and exclusion criteria were included in this study.

Table 1. The baseline characteristics of the study subjects

\begin{tabular}{|c|c|}
\hline Characteristics & \\
\hline \multicolumn{2}{|l|}{ Sex } \\
\hline Male (n/\%) & $52(57.8)$ \\
\hline Female (n/\%) & $38(42.2)$ \\
\hline Age (mean $\pm S D$ ) years-old & $48.83 \pm 12.8$ \\
\hline HD duration, median (min-max) months & $36(6-228)$ \\
\hline $\begin{array}{l}\text { Previous history of illnesses } \\
\text { Diabetes mellitus (n/\%) } \\
\text { Hypertension (n/\%) } \\
\text { Stroke (n/\%) } \\
\text { Cardiac disease }(n / \%)\end{array}$ & $\begin{array}{l}26(28.9) \\
74(82.2) \\
9(10) \\
16(17.7)\end{array}$ \\
\hline Smoking History (n/\%) & $6(6.7)$ \\
\hline \multicolumn{2}{|l|}{ Laboratorium assessments } \\
\hline a. BUN (mean $\pm S D) \mathrm{mg} / \mathrm{dL}$ & $58.33 \pm 14.4$ \\
\hline b. Creatinine (mean $\pm S D$ ) $\mathrm{mg} / \mathrm{dL}$ & $10.77 \pm 2.7$ \\
\hline c. Albumine $(\mathrm{mg} / \mathrm{dL})$ & $4.02 \pm 0.3$ \\
\hline d. Hemoglobin $(\mathrm{g} / \mathrm{dL})$ & $9.44 \pm 1.6$ \\
\hline e. Leukocyte count, (mean $\pm \mathrm{SD}) \times 10^{3}$ cell/回 & $6.97 \pm 1.7$ \\
\hline 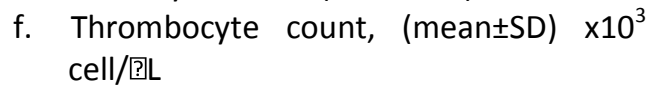 & $\begin{array}{l}217.7 \pm 55.9 \\
0.24\end{array}$ \\
\hline $\begin{array}{l}\text { g. C-reactive protein (CRP), median } \\
\text { min-max) } \mathrm{mg} / \mathrm{L}\end{array}$ & $\begin{array}{l}(0.2-9.9) \\
11.89\end{array}$ \\
\hline h. IL-6, median (min-max) pg/dL & $\begin{array}{l}(1.32-87.53) \\
1.42\end{array}$ \\
\hline IL-1 $\beta$, median (min-max) pg/dL & $(0.1-16.0)$ \\
\hline IL-10, median (min-max) pg/dL & $\begin{array}{l}4.15 \\
(1.46-55.5)\end{array}$ \\
\hline
\end{tabular}


A total of 90 subjects who had fulfilled the inclusion and exclusion criteria were included in this study. The baseline characteristics of those subjects were presented in Table 1.

The mean age of subjects in this study was $50.58 \pm 13.1$ years-old. This result was similar with a previous study in India which showed that the mean age of subjects was $49.75 \pm 13.84$ years-old, also with Kamel study in Kairo that showed the mean age for ESRD patients of $52.8 \pm 8.8$ years-old. ${ }^{6,7}$ The percentage of male patients was found dominant during this study, this was parallel with the study conducted by Hendrix et al., ${ }^{8}$ in the Netherlands with $67.5 \%$ of male ESRD patients. ${ }^{8}$ The median of HD duration in this study was 36 months. The HD duration in this study was longer than the study was conducted by Hendrix et al., ${ }^{8}$ in which the median of HD duration was 20 months. ${ }^{8}$

In this study, we tracked the related-history of previous illnesses included hypertension, stroke, diabetes and cardiac diseases. Total subjects who had a risk factors for hypertension was $82.2 \%$. This number was lower compared with the results of a study conducted by Jeli in Serbia (88\%). ${ }^{9}$ Hypertension is one of risk factor for chronic renal disease, this maybe because a severe and uncontrolled hypertension will cause changes in arterioles vessel walls (arteriol fibrinoid sclerosis) and will impair the renal function, on the other side, hypertension may also be an impact from chronic renal disease (renal hypertension). ${ }^{9}$

There was a total of $28.9 \%$ of subjects who had risk factor for diabetes mellitus. One of target organ for DM complications is the kidney. This is began with DM nephropathy that if continued and uncontrolled will cause chronic kidney failure. ${ }^{9}$ Blood Urea Nitogen (BUN) and creatinine levels preHD were found to be $49.49 \pm 15.6 \mathrm{mg} / \mathrm{dL}$ and $10.77 \pm 2.7 \mathrm{mg} / \mathrm{dL}$, respectively. The mean of creatinine level in this study was higher than a previous study by Krishnamurthy ${ }^{7}$ which showed a creatinine level of $7.22 \pm 2.22 \mathrm{mg} / \mathrm{dL}$. The mean of albumine level and leukocyte counts were found in the normal range. The role of leukocyte assessment was to exclude infections and the role of albumin assessment was to reflect the liver synthesis function. ${ }^{7}$ Liver function failure will cause the disruption of CRP inflammatory marker synthesis. There were correlations between IL-1 $\beta \alpha v \delta$ IL-10, IL -6 and CRP levels as shown in Figure 1 and 2 . The IL
IL-1b level was not found to be correlated with CRP level $(p=0.337)$. There were correlations between IL-1b and IL-10, IL-6 and CRP levels as shown in Figure 1 and 2 . The IL-1b level was not found to be correlated with CRP level $(p=0.337)$.

Interleukin-1b was found to be weakly correlated with IL-10 (IL-10 an anti-inflammatory cytokine). Interleukin-1 is an interleukin that takes part in inflammatory response and has an important role in kidney recovery process after ischemic conditions, such as in renal fibrosis. The infiltration of immune cells and other factors played a role in the fibrogenesis process of kidney. Some animal studies showed that IL1 and IL- 6 levels in animals with ESRD and renal fibrosis were higher than normal levels. ${ }^{10}$ The weak correlation between IL-1b and IL-10 was because IL-10 secretion always followed the proinflammatory factors. All factors that triggered proinflammatory cytokines secretion will trigger the release of anti-inflammatory cytokines, the consequences of anti-inflammatory cytokines secretion will happen minutes after the proinflammatory cytokines are released. This explained a positive correlation between the level of proinflammatory and anti-inflammatory cytokines. ${ }^{11}$

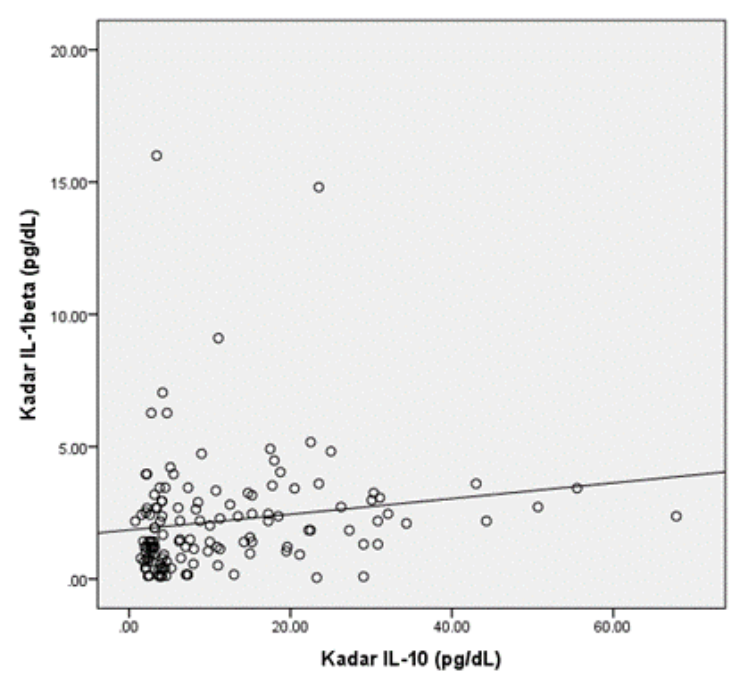

Figure 1. Correlation between IL-1b and IL-10 levels $(r=0.302, p=0.001)$

Interleukin 10 that was produced by some subpopulation of T cells, such as Th2, regulatory T cells, Natural Killer (NK) cells and some other cell types such as macrophages, dendritic cells, B lymphocyte cells and mesangial also endothelial (kidney) cells will. in turn $d$ - 
o some actions that will suppress the inflammatory responseone of them is by inhibiting the production of cytokines IL-1 and TNF a from activated monocyte cells. Interleukin 10 also has a role in suppressing chemokines that will attract leukocytes to the inflammation areas. ${ }^{11-13}$

Interleukin $1 \mathrm{~b}$ also had a weak correlation

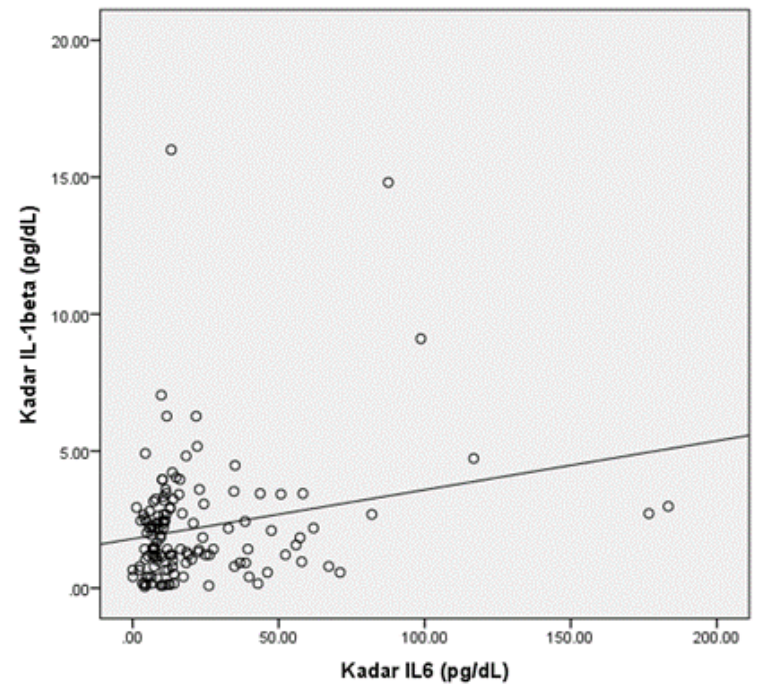

Figure 2. Correlation between IL-1b and IL-6 levels ( $r=0.232$, $p=0.029$ )

with IL-6 ( $r=0.229, p=0.012)$ but it did not correlate with CRP. This was because of IL-1b one of the natural stimulants that functions triggering the IL- 6 secretion. ${ }^{14}$ Interleukin-6 a cytokine that was produced by various cell types in various conditions such as infection, trauma and immunologic disturbance. The functions of IL- 6 are to trigger and activate $T$ cells, $T$ cell activation, $B$ cells differentiation and in the induction of acute phase proteins by hepatocytes. ${ }^{15}$ Among the other proinflammatory cytokines, IL-6 and soluble IL-6 receptors are considered as the central regulators of inflammation processes.

In this study, the IL-1b level was found not to be correlated with CRP level ( $p=0.337)$. C-reactive protein is an acute phase protein that was produced by liver as a response for systemic cytokinemia (interleukin-6, interleukin-1, tumor necrosis factoralpha) condition during tissues injury, infection and inflammation. ${ }^{16}$ C-Reactive Protein (CRP) secretion was more influenced by IL- 6 and was indirectly influenced by IL- $1 \mathrm{~b}$ therefore IL- $1 \mathrm{~b}$ was not directly correlated with CRP. Linkage between CRP and IL-1b happened undirectly, IL-1b will trigger IL-6 secretion secretion and in turn, IL- 6 will trigger CRP secretion by hepatocytes. ${ }^{16}$

It was also found that IL-10 had a moderate positive correlation with IL-6 $(r=0.418, p=0.001)$. This result was different with the study by Girndt et al., ${ }^{17}$ which showed a moderate negative correlation between IL-10 secretion and IL- 6 level $\left(r^{2}=0.54, p<0.01\right)$ in the ESRD subjects group who had a good response to vaccination. In patients with poor response to vaccination, no correlation was found between IL-10 and IL-6 levels. ${ }^{17}$

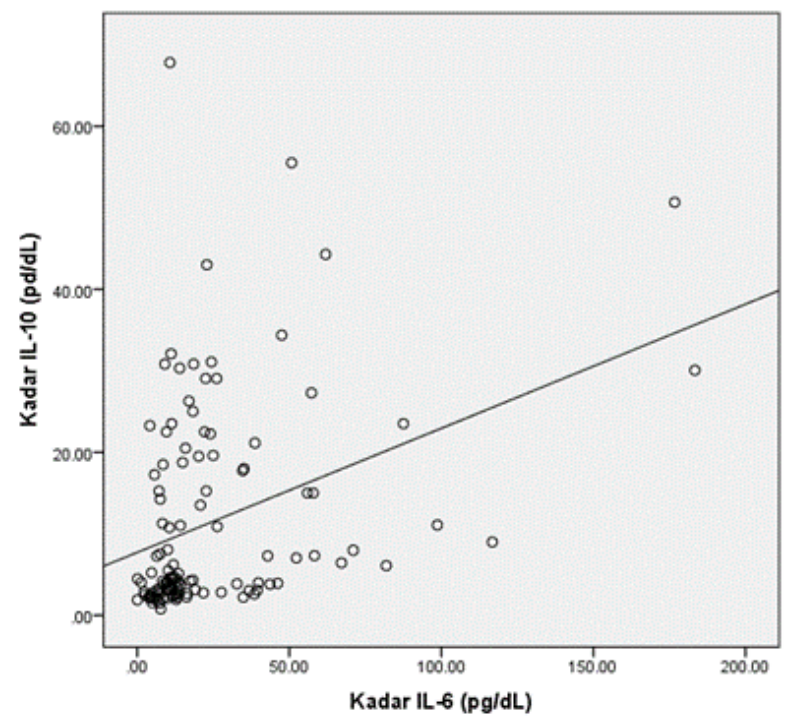

Figure 3. Correlation between IL-6 and IL-10 levels ( $r=0.418$, $p=0.001)$.

The positive correlation that was found in this study might be because anything that could trigger the secretion of proinflammatory mediators will trigger anti-inflammatory mediators secretion and one of them was IL-10, therefore the secretion of IL- 6 and IL10 happened in parallel. IL-10 secretion always followed the proinflammatory factors. In the next stage, IL-10 secretion will suppress the proinflammatory cytokines, such as IL-6. In this study, that stage might be not achieved yet. Another consideration was IL- 6 and IL-10 excreted together in the kidney, therefore in patients with renal function disorders there will be an elimination disturbance of those cytokines, therefore the level of these cytokines in the circulation will be equally high.

Another factor influencing this balance condition is the genetic factor. A study conducted by Machananda et al., showed that there was an interindividual genetic variation in the capability of IL-10 secretion, a 
group included in the high IL-10 producer genotype (alel G), also groups that have a low IL-10 secretion capability (genotype $A / A /$, gene polymorphism IL-10 -1082). This variation will influence the balance condition between inflammatory and antiinflammatory cytokines. ${ }^{14,18}$ Genetic factors also may influence the IL-6 secretion. A study conducted by Okada showed that IL-6 secretion was also influenced by genetic factors. IL6-57266 genotype secreted lower IL- 6 levels than other genotypes. ${ }^{20}$

Another factor that caused the difference between this study and a study by Girndt ${ }^{17}$ was the cytokines source that has been examined. In this study, the measured cytokines were plasma cytokines but in the study conducted by Girndt ${ }^{17}$, the measured cytokines were came from cytokine secretion produced by Peripheral Blood Mononuclear Cell (PBMC) cultures. ${ }^{17}$

Another study parallel with this study was a study by Kamel. ${ }^{6}$ Kamel et al. proved that there was a correlation between proinflammatory cytokine (IFNg) and anti-inflammatory cytokine (IL-10) production within endothelial dysfunction condition was assessed from Soluble Intercellular Adhesion Molecule (SICAM) and soluble E-selectin (SEselectin) biomarkers in DM type-2 patients who had nephropathy diabetic and ESRD. The results of that study showed that there was a correlation between IFN $g$ and IL-10 level with $r=0.444(p<0.01) .^{6}$

The IL-10 level in this study was found to have a weak positive correlation with CRP level. This was quite different from the study by El Shamma ${ }^{20}$ in pediatric patients who underwent hemodialysis, either with good or poor venous access. In that study, a weak negative correlation was found between IL-10 and CRP levels ( $r=-0.36 ; p=0.001$ ). Patients with poor venous access (AV shunt) were found to have higher CRP level compared with the good venous access group. ${ }^{20}$ In this study, all subjects had a good venous access. The weak positive correlation was found in this study was because IL-10 secretion always followed the secretion of proinflammatory mediators, such as CRP.

There was a significant moderate positive correlation between IL- 6 and CRP levels ( $r=0.534$, $\mathrm{p}=0.001$ ). Interleukin- 6 is a cytokine produced by various cells in various conditions such as infection, trauma and immunologic disturbances. IL- 6 acts by trigger and activate $T$ cells, B cells differentiation and in the acute phase proteins induction by hepatocyte trigger and activate T cells, B cells differentiation and in the acute phase proteins induction by hepatocyte cells, such as CRP. ${ }^{21}$

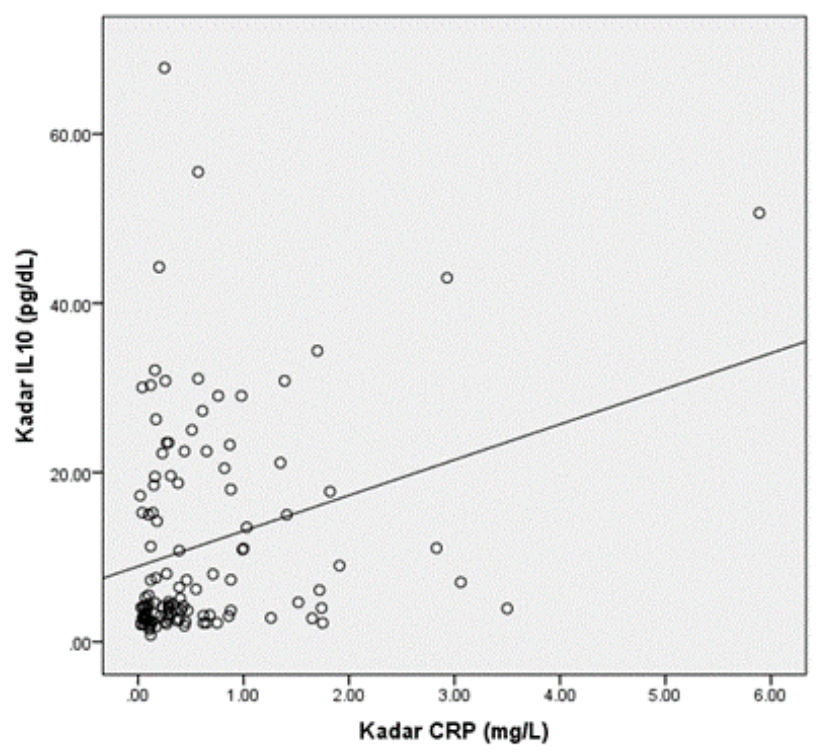

Figure 4. Correlation between IL-10 and CRP levels ( $r=0.295$, $p=0.005$ )

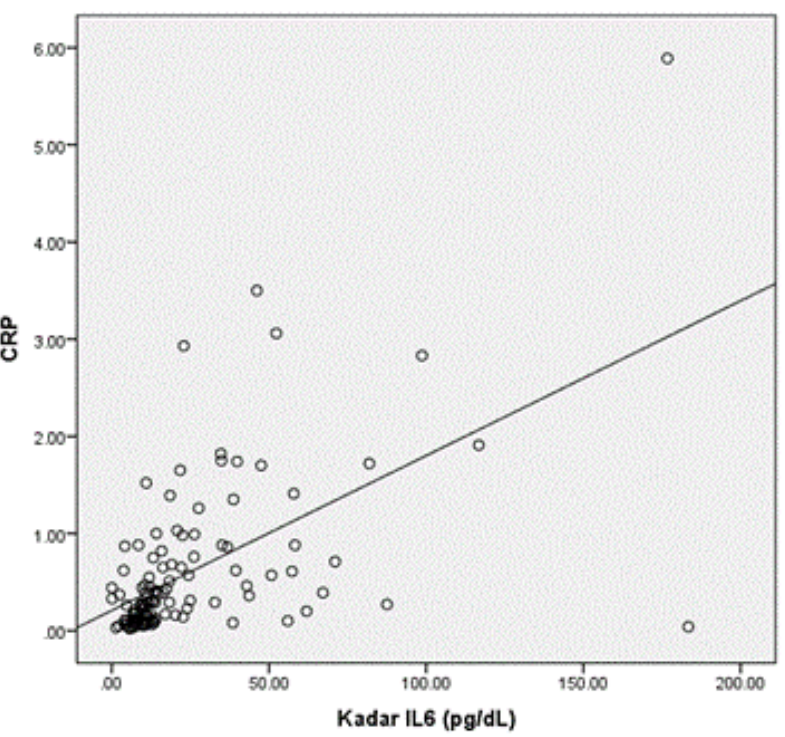

Figure 5. Correlation between IL-6 and CRP levels ( $r=0.534$, $p=0.001$ )

\section{CONCLUSION AND SUGGESTION}

There were significant weak correlations between IL$1 \mathrm{~b}$ and IL-1b and IL-6, also between IL-6 and CRP. Moderate correlations were found between IL-1b and IL- 6 also between IL- 6 and CRP. There was no significant correlation found between CRP level and IL6 and IL-1ㅁ․ 
Further study of cytokine secretion-related polymorphism is warranted.

\section{REFERENCES}

1. Coresh J, Selvin E, Stevens LA. Prevalence of chronic kidney disease in the United States. JAMA 2007; 298(17): 2038-47.

2. Nagane NS, Ganu JV, Jagtap PE. Study of oxidative stress in pre- and post-hemodialysis in chronic renal failure patients. Biomed Research 2013; 24(4): 498502.

3. Brown MD, Feairheller DL, Thakkar $\mathrm{S}$, Veerabhadrappa, Park JY. Racial differences in tumor necrosis factors a-indiced endothelial microparticles and IL-6 production. Vascular Health and Risk Management 2011; 7: 541-550.

4. Tripathi G, Rangaswamy D, Borkar M, Prasad N, Sharma RK, Sankhwar SN, Agrawal S. Interleukin-1 gene cluster variants in hemodialysis patients with end stage renal disease: An association and metaanalysis. Indian Journal of Nephrology 2015; 25(1): 34-42.

5. Nand N, Aggarwal HK, Yadav RK, Gupta A, Sharma M. Role of high-sensitivity C-reactive Protein as a Marker of Inflammation in pre-dialysis patients of chronic renal failure. JIACM 2009; 10(1 \& 2): 18-22.

6. Kamel L, Morsy A, El Shamaa A. T-cell cytokine production and endothelial dysfunction in type 2 diabetic patients with nephropathy. Eastern Mediterranean Health Jour 2009; 15(4): 807-16.

7. Krishnamurthy $\mathrm{N}$, Arugasany K, Anand U, Anand CV, Aruna V, Venu G, Gayathri R. Effect of hemodialysis on circulating cystatin $\mathrm{c}$ levels in patients with end stage renal disease. Indian Jour of Clin Biochem 2010; 25(1): 43-46.

8. Hendrikx TS, Van Gurp EA, Mol WM, Schoordijk W, Sewgobind VDK, ljzermans JNM, Weimar W, Baa CC. End-stage renal failure and regulatory activities of $\mathrm{CD}^{+}{ }^{\mathrm{CD} 25}{ }^{\text {bright+ }^{\circ} \text { FoxP3 }}{ }^{+} \mathrm{T}$ cells, Nephrol Dial Transplant 2009; 4: 1969-78.

9. Jeli $M$, Cvetkovi T, Djordjevi V, Damnjanovi G, Vlahovi $P$, Koci $G$, et al. Hepcidin and iron metabolism disorders in patients with chronic kidney disease. Vojnosanit Pregl 2013; 70(4): 368-373.
10. Papanicolaou DA, Wilder RL, Manolagas SC, Chrousos GP. The pathophysiologic roles of interleukin- 6 in human disease. Ann Intern Med 1998; 128(2): 127-137.

11. Sabat R. IL-10 family of cytokines. Cytokine Growth Factor Rev 2010; 21(5): 315-24.

12. Nordfors L, Lindholm B, Stenvinkel P. End-stage renal disease--not an equal opportunity disease: the role of genetic polymorphisms. J Intern Med 2005; 258(1): 112.

13. Stevinkel $P$, Ketteler M, Johnson RJ, Lindholm B, Pecoits R, Riella M, Heimburger O, Cederhom T, Girndt M. IL-10, IL-6 and TNFa: Central factors in the altered cytokine network of uremia-The good, the bad and the ugly. Kidney International 2005; 67(4): 1216-1233.

14. Yhee JY, Yu CH, Kim JH, Sur JH. Effects of T lymphocytes, interleukin-1 and interleukin-6 on renal fibrosis in canine end-stage renal disease. J Vet Diagn Invest 2008; 20(5): 585-92.

15. Niskane A, Saramäki M, Schwandt E. Myeloperoxidase (MPO) and hs-CRP as predictive factors for myocardial infarction. CLI, 2006; 7: 38-9.

16. Girndt $M$, Kohler $H$, Weick ES, Schlaak JF, Meyer KH, Fleisher B. Production of interleukin-6, tumor necrosis factor a and interleukin 10 in vitro correlates with the clinics; immune defect in chronic hemodialysis patients, Kidney Int. 1995; 47: 559-65

17. Manchanda PK, Singh R, Mittal RD. Cytokine (IL-10 _1082 and_819) and chemokine receptor (CCR2 and CCR5) gene polymorphism in north indian patients with end-stage renal disease. DNA and Cell Biology 2009; 28 (4): 177-83.

18. Okada R, Wakai K, Naito M, Morita E, Kawai S, Hamajima N, Takashima N. Pro-/anti-inflammatory cytokine gene polymorphisms and chronic kidney disease: a cross -sectional study. BMC Nephrology 2012; 13(2): 1-8.

19. Elshama MF, Sabry S, Galal A, Koura H, Kantoush N, Rasheed M, Thabe EH. Serum interleukin 10 levels in vascular access failure in Egyptian children on hemodialysis. J Clin Basic Cardiol 2009; 12(1): 18-23.

20. Papanicolaou DA, Wilder RL, Manolagas SC, Chrousos GP. The pathophysiologic roles of interleukin- 6 in human disease. Ann Intern Med 1998; 128(2): 127-137. 